\title{
REDESIGN OF MANUFACTURING PROCESS FOR GEAR TRANSMISSION BOX COMPONENT OF HAND TRACTOR
}

\author{
Moses L. Singgih and Ahmad Fatih Fudhla \\ Department of Industrial Engineering \\ Institut Teknologi Sepuluh Nopember \\ Kampus ITS Keputih Sukolilo, Surabaya 60111 INDONESIA \\ e-mail: moses@ie.its.ac.id ; ahmadfatihfudhla@gmail.com
}

\begin{abstract}
Design for Manufacture (DfM) is one of approach in product design development. It is integration of art design, material and process choosing in quality consideration. Gear Transmission Box (GTB) component of hand tractor is one of main component that compile hand tractor. It's base component, other components are connected to it. It was produced in combination of casting-machining process and its manufacturing cost is the second highest that cause high production cost. To solve it, development or redesign of fabrication is needed. To realize it, load and force it detained have to be known, and than create alternatives design and process using DfM approach that yield cheaper design but still strong enough for detaining operation load. In this research, two alternatives were resulted. Both are using St 37 plate as base material and combination plate metal forming, welding and machining as basic process. The First redesign reduces manufacturing cost $11.75 \%$, and the second $27 \%$. From this research, it can be concluded that in design stage, there are a lot of potential things can be improved and developed that make production cheaper.
\end{abstract}

Key words : Design for manufacture, manufacturing cost, load analysis

\section{INTRODUCTION}

Currently, the industrial competition becomes more complex. The companies compete each other by relying on their own potential to get and conquer the market. This condition pushed them to do continuous improvement for their product that more user/costumer friendly in material, weight or technology offered the price that could be reached by customer. The main contributor of manufacture cost is design factor. In fact, quite often more than $80 \%$ of the manufacturing cost of a product is determined by this factor, yet manufacturing is not involved (Anderson 2007).

One of the approach methods in product design development is Design for Manufacture (DfM). DfM is a systematic and comprehensive design approach that considering material, manufacture method, design process, assembly, testing and quality.

In agricultural world, Farm processing plant in a short time will improve the productivity. One of needing in farm processing is agricultural equipment mechanized. In Indonesia, One other have there is hand tractor. But it will become the barrier when price of hand tractor on farmer is too high. One of cause is the level of production cost. It was happen in hand tractor product of PT Agrindo one of notable hand tractor producer in Indonesia. Based on their analysis, Gear
Transmission Box (GTB) is one of main factor which increase production cost. It is the second highest of manufacturing cost from seven components. Gear transmission box is produced using casting process. It can't be produced in mass production. So its process and design must be developed.

By Using DfM concept, in this paper will be shown the process of GTB design development. Here, loads that GTB detained are calculated and become main consideration as a quality parameter. The best design is the cheaper design.

\section{PRODUCT DEVELOPMENT DESIGN CONCEPT}

\section{DfM Definition}

There are some definitions about Design for Manufacture (DfM). Kalpakjian (1989) define it as a comprehensive approach to produce things and integrate design process with choosing material and manufacturing method, process planning, machine and tools. And it was defined more by Anderson (2007). He defined it as the process of proactively designing products to (1) optimize all the manufacturing functions: fabrication, assembly, test, procurement, shipping, delivery, service, and repair, and (2) assure the best cost, 
quality, reliability, regulatory compliance, safety, time-to-market, and customer satisfaction.

Based on definitions above, in a few word Design for Manufacture is integration of art design, material and process choosing in quality consideration

\section{DfM Concept}

From a manufacturability perspective, DFM approach help avoid the "disconnect" that often occurs when the design team puts forth a product that cannot be manufactured. DFM benefits the design team by allowing them to explore alternatives in processes and materials, while showing the cost impact of each decision. This allows designers to improve the manufacturability of their product through simplification.(Andrews, 2006)

Marginally Ulrich and Eppinger (2000) said that there are five step in running DfM approach;

1. Approximating manufacturing cost

There are three category of manufacturing cost, they are component cost, assembly cost and overhead cost.

2. Lessening component cost

There are Some chance that can be applied to lessening cost; simplification part design, allow not so good surface refining, using cheaper material, using more efficient machine, tools and control, etc.

3. Lessening assembling cost

Assembly cost can be calculated based on labor cost and processing cost which is quantifying from assembly material cost and tooling cost.

4. Lessening overhead cost.

It can be done by minimize systemic complexity.

5. Considering influence of decision DfM to another factors

Factors that have to be known are; development time, development cost, product quality, the external factors including reuse component and life time cost.

\section{Load Analysis}

Load analysis is used to know how much and how big force/load that has to be shored up. It is use full in measuring design strength.

Eccentrically loaded rivets

Making use of law of static, which says that a force may be moved to a parallel coplanar position by replacing it by an equivalent force and couple, $F$ (solid line) is replaced by the equivalent force $F$ (dotted), acting through the center of gravity of rivet group, and equivalent couple $\mathrm{Fe}$ like shown in. $F($ dotted) are apportionment to each rivet given by,

$$
F_{1}(\text { dotted })=F_{2}(\text { dotted })=F_{n}(\text { dotted })=\frac{F}{n}
$$

And Forces $\left(T_{j}\right)$ caused by couple $F e$, given by

$$
T_{j}=\frac{\text { Fed }_{j}}{\sum_{k=1}^{n} d_{k}^{2}}
$$

Where $e$ is horizontal distance to center of gravity of rivet system in meter, $F$ is eccentirc force that causes load in rivets systems in newton and $d_{k}$ is variable that show distance each rivet to center of gravity of rivet systems in meter.

Load in bearing caused gear and shaft rotary

Load in bearing that caused by power transmitted consist of two forces. Tangential force $\left(F_{t}\right)$ and radial force $\left(F_{r}\right)$ (Deutchman, 1975). Tangential direction is go to center of bearing but radial force direction is opposite with radial movement. Both are given by

$F_{r}=F_{t} \tan \Phi$

$F_{t}=\frac{(h p)(33000)}{V_{p}}$

And Pitch velocity $\left(V_{p}\right)$ is given by

$$
V_{p}=\frac{\pi d n}{12}
$$

Torsion $(T)$ And power that transmitted $(h p)$ given by

$$
h p=\frac{T n}{63000}
$$

Where $\Phi$ is pressure angle of gear in degree, $h p$ is power transmitted by gear in horse power, torsion $(T)$ in inches-pound, angular velocity $n$ in rpm, gear diameter $d$ in inches, $V_{p}$ in $\mathrm{ft} / \mathrm{min}, F_{t}$ and $F_{r}$ in lbf.

\section{Machining}

\section{Cutting time of freis process}

In his book, Taufiq (1993) was explain that cutting time $\left(t_{c}, \min \right)$ is depend on length of cutting track $\left(l_{t}, \mathrm{~mm}\right)$ and cutting speed $\left(v_{f}, \mathrm{~mm} / \mathrm{min}\right)$ that given by,

$t_{c}=l_{t} / v_{f}$

$l_{t}=l_{v}+l_{w}+l_{n}$

where $l_{v}$ is starting track in $\mathrm{mm}$ and $l_{n}$ is finishing track in $\mathrm{mm}$

$$
l_{v} \geq \sqrt{a(d-a)} \text {; for horizontal freis }
$$


$l_{v} \geq 0$; for vertical freis

$l_{n} \geq 0$; for horizontal freis

$l_{n}=d / 2 ;$ for vertical freis

Cutting time of drilling and boring process

Similar with freis process, Taufiq (1993) was explain about cutting time of drilling and boring process is given by

feeding speed $\left(f_{z}, \mathrm{~mm} /\right.$ rotation $)$ for each tool

$f_{z}=v_{f} /(n . z) ; z=2$

Cutting time $\left(t_{c}, \min \right) 4$.:

$t_{c}=l_{t} / v_{f}$

$l_{t}=l_{v}+l_{w}+l_{n}$

Where $v_{f}$ is cutting speed in $\mathrm{mm} / \mathrm{min}, n$ is number of tools, $l_{v}$ is starting track in $\mathrm{mm}$ and $l_{n}$ is finishing track in $\mathrm{mm}$

Machining total operation time

for each machining process, machining total operation can be found by adding non productive time $\left(t_{a}\right)$, tool changing time $\left(t_{d}\right)$ and cutting time $\left(t_{c}\right)$.

$t_{o}=t_{a}+t_{d}+t_{c}$

\section{Force Needed in Pressing Process}

Force needed for percing and blanking $\left(F_{s}\right)$ some plat material that have cutting periphery $L_{s}$ and thickness $S_{o}$ are given by:

$F_{s}=L_{s} \cdot S_{o} \cdot K_{s}$

(PN RAO, Manufacturing Technology)

Where $K_{s}$ is shift tension that is gotten from ultimate strength of material $\left\{0,8 \sigma_{u}\right\}\left(\mathrm{kg} / \mathrm{mm}^{2}\right)$

\section{Minimum Radius $\left(\mathbf{R}_{\min }\right)$ in Drawing Process}

Necking criteria

This Necking causes dilution of part of metal at bent area. Necking happen when elongation or lengthening in external fiber of specimen $e_{t}$ that exceeding the uniform elongation of material $e_{u}$ from unaxial tensile test.

$e_{t}=\frac{1}{\left(\frac{2 . R}{s_{o}}\right)} \leq e_{u}$

So, minimum radius $R_{\text {min }}$ can be known by

$R_{\min }=\frac{s_{0}}{2}\left(\frac{1}{e_{u}}-1\right)$

Where $\mathrm{R}$ is bending radius. $s_{o}$ is material thickness. And $e_{u}$ can be calculated from $e_{u}=$ $(\exp . n-1) . n$ is got from tensile test.
Cracking criteria.

Cracking cause the rip at bent area. The following Equation determined by $\mathrm{q}$ that is reduction of longitudinal section of material that resulted by $\mathrm{f}$ ] unaxial tensile test.

For less ductile material, $\mathrm{q}<0,2$

$R_{\min }=s_{o} \times\left(\frac{1}{2 . q}-1\right)$

For ductile material, $\mathrm{q}>0,2$

$R_{\min }=s_{o} \times \frac{(1-q)^{2}}{\left(2 . q-q^{2}\right)}$

$\underline{\text { Strain factor }}$

Good drawing design has to pass strain factor. strain of design $e_{s}$ must be less than elongation of material $e_{u}$ from unaxial tensile test.

$e_{s}=\frac{1-\cos \alpha}{\frac{c}{w} \pm 1} \leq e_{u}$

Where $\alpha$ is bending angle. $c$ is inner radius of bending line. $w$ is flange width.

\section{REDESIGN PROCESS \\ Initial Design}

Initial GTB consist of two part, left hand side (GTB LH) and right hand side (GTB RH). It is shown in Figure 1.

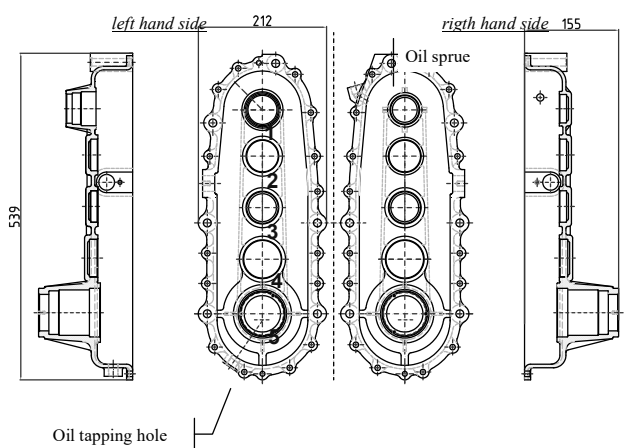

Figure 1. Initial gear transmission box

GTB are produced in combination casting and machining process. It mean after casting finished, GTB should be entered seven machining step. Process sequences GTB RH and LH are shown in figure 2 and 3

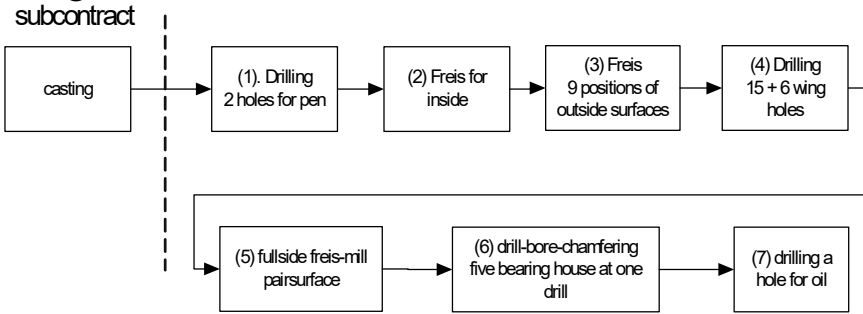

Figure 2. Flow Process Diagram of GTB RH 


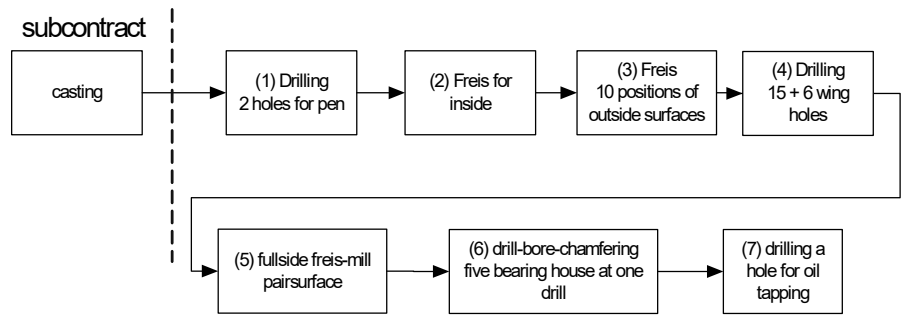

Figure 3. Flow Process Diagram of GTB LH

The using of machine and each cost rate are shown in Table 1. process can be seen in figure 2 and 3 .

Table 1. using and cost rate of machine

\begin{tabular}{|l|c|c|c|}
\hline \multicolumn{1}{|c|}{ machine } & Type & Process & cost rate (Rp/min) \\
\hline vertical milling & & 5 & 24.1 \\
\hline horizontal milling & $1 \mathrm{a}$ & 2 & 50.1 \\
\cline { 2 - 4 } & $1 \mathrm{~b}$ & 3 & 50.1 \\
\cline { 2 - 4 } & $2 \mathrm{a}$ & $6(\mathrm{RH})$ & 50.1 \\
\cline { 2 - 4 } & $2 \mathrm{~b}$ & $6(\mathrm{LH})$ & 50.1 \\
\hline \multirow{4}{*}{ drilling machine } & Standard1 & 1 & 37.1 \\
\cline { 2 - 4 } & Standard 2 & 4 & 37.1 \\
\cline { 2 - 4 } & Radial & 7 & 37.1 \\
\hline
\end{tabular}

Manufacturing Cost

All of initial manufacturing costs were got from the company. Overhead cost decided by PT Agrindo $5 \%$ from material cost and 10\% from machine, assembly and labor cost. Tabulation of manufacturing cost of initial GTB is shown in Table 2. assembly cost is zero because in this initial design there aren't assembly operation;

Table 2. Manufacture cost of initial GTB (Rp)

\begin{tabular}{|l|r|r|r|r|r|r|}
\hline part name & material cost & $\begin{array}{c}\text { machine } \\
\text { cost }\end{array}$ & $\begin{array}{c}\text { labour } \\
\text { cost }\end{array}$ & $\begin{array}{c}\text { assembly } \\
\text { cost }\end{array}$ & $\begin{array}{c}\text { overhead } \\
\text { cost }\end{array}$ & $\begin{array}{c}\text { manufacturing } \\
\text { cost }\end{array}$ \\
\hline GTB LH & $144,500.0$ & $11,149.3$ & $30,917.3$ & 0.0 & $11,431.7$ & $197,998.2$ \\
\hline GTB RH & $143,500.0$ & $9,229.0$ & $26,224.2$ & 0.0 & $10,720.3$ & $189,673.5$ \\
\hline total & $288,000.0$ & $20,378.3$ & $57,141.5$ & 0.0 & $22,152.0$ & $387,671.7$ \\
\hline
\end{tabular}

\section{Load Analysis}

GTB detain loads from another main component of hand tractor. There are two kind of load, static and dynamic. Static loads are caused by weight of another component. There are Frame handle $22 \mathrm{~kg}$, Frame engine dan engine $116 \mathrm{~kg}$ Rear hitch and plow $34 \mathrm{~kg}$, floating wheel $52 \mathrm{~kg}$ and GTB their self $36 \mathrm{~kg}$. dynamics by rotation of gear systems. Maximum load is the biggest resultant of dynamic and static load. By equation (1), (2), (3), (4), (5) and (6). Maximum load can be calculated. It is $57.39 \mathrm{kN}$ in GTB RH.

\section{Design 1}

This design use ST 37 plate as material. Thickness of main body is $6 \mathrm{~mm}$, main shaft cover and pulley shaft cover are $4.3 \mathrm{~mm}$, secondary shaft is $2 \mathrm{~mm}$. this design consist of 13 part each side. (shown in Figure 4)

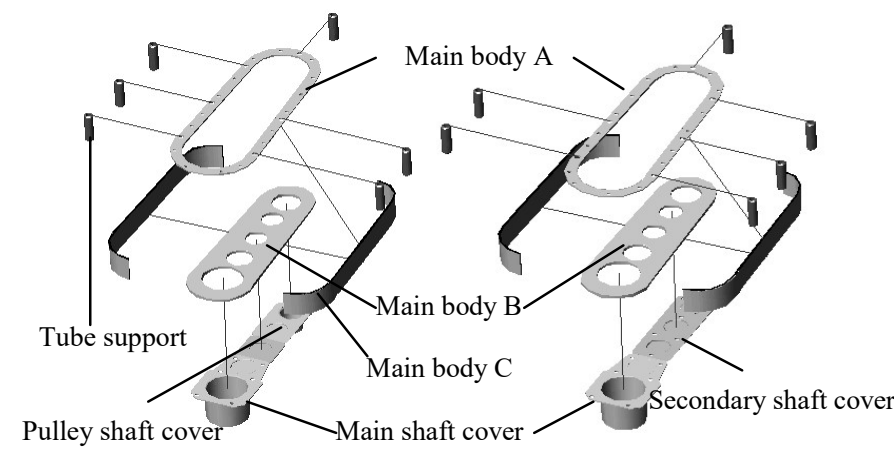

Figure 4. Design 1 of GTB breakdown

Design 1 is produced in combination plate metal forming (drawing, piercing, shearing, banding and blanking), welding and machining. These operation were shown in Table 3 and 4. machine cost was calculated with multiply the machine cost rate (table 1) with machine operation time. Labour cost was got by multiply labour time with the labour cost rate $(\mathrm{Rp} 122.45)$.

Table 3. Production operation list of design 1

\begin{tabular}{|c|c|c|c|}
\hline \multicolumn{4}{|c|}{ Gear Transmission Box LH } \\
\hline Operation & Machine & $\begin{array}{l}\text { Machine } \\
\text { cost (Rp) }\end{array}$ & $\begin{array}{c}\text { labour } \\
\text { cost (Rp) }\end{array}$ \\
\hline main body forming ( $\mathrm{A}$ and $\mathrm{B})$ & hidrolic pressure & 6552.9 & 2371.9 \\
\hline main body forming ( $\mathrm{C}$ ) & hidrolic pressure & 8586.1 & 3107.8 \\
\hline main shaft cover forming & hidrolic pressure & 4773.4 & 1727.8 \\
\hline secondary shaft cover forming & hidrolic pressure & 4330.2 & 1567.4 \\
\hline pulley shaft cofer forming & hidrolic pressure & 4712.5 & 1705.7 \\
\hline tube support cutting & band SAW Mega & 659.3 & 2755.1 \\
\hline Full side freis mill & vertical milling & 1903.4 & 5167.4 \\
\hline Drilling 2 pen holes & standard 1 drilling & 827.4 & 2730.6 \\
\hline Boring+chamfering 5 holes together & horizontal 2 milling & 456.4 & 1115.5 \\
\hline Drilling oil taping & radial drilling & 217.1 & 716.3 \\
\hline Drilling oil sprue & radial drilling & 217.1 & 716.3 \\
\hline Drilling 8 holes $\varnothing 7$ & standard 1 drilling & 280.1 & 924.5 \\
\hline Drilling 6 holes $\emptyset 8$ & standard 2 drilling & 254.2 & 838.8 \\
\hline Drilling 6 holes $\emptyset 10$ & standard 1 drilling & 253.8 & 837.6 \\
\hline Drilling 15 holes $\emptyset 8$ & standard 2 drilling & 369.9 & 1220.8 \\
\hline freis main shaft cover & horizontal 2 milling & 314.7 & 769.0 \\
\hline freis pulley shaft cover & horizontal 2 milling & 296.1 & 723.7 \\
\hline drill 6 holes main shaft cover & standard 2 drilling & 252.3 & 832.7 \\
\hline drilling 3 holes secondary shaft cover & standard 1 drilling & 213.3 & 704.1 \\
\hline Drilling 6 holes pulley shaft cover & standard 2 drilling & 252.3 & 832.7 \\
\hline \multicolumn{2}{|l|}{ LH-Total } & 35722.5 & 31365.6 \\
\hline \multicolumn{4}{|c|}{ Gear Transmission Box RH } \\
\hline Operation & Machine & $\begin{array}{l}\text { Machine } \\
\text { cost }(\mathrm{Rp})\end{array}$ & $\begin{array}{c}\text { labour } \\
\text { cost (Rp) } \\
\end{array}$ \\
\hline main body forming ( $\mathrm{A}$ and $\mathrm{B})$ & hidrolic pressure & 6552.9 & 2371.9 \\
\hline main body forming $(\mathrm{C})$ & hidrolic pressure & 8586.1 & 3107.8 \\
\hline main shaft cover forming & hidrolic pressure & 4773.4 & 1727.8 \\
\hline secondary shaft cover $(\mathrm{RH})$ forming & hidrolic pressure & 4621.2 & 1672.7 \\
\hline tube support cutting & band SAW Mega & 659.3 & 2755.1 \\
\hline Full side freis mill & vertical milling & 1903.4 & 5167.4 \\
\hline Drilling 2 pen holes & standard 1 drilling & 827.4 & 2730.6 \\
\hline Boring + chamfering 5 holes together & horizontal 2 milling & 456.4 & 1115.5 \\
\hline Drilling 6 holes $\varnothing 7$ & standard 1 drilling & 254.2 & 838.8 \\
\hline Drilling 6 holes $\emptyset 8$ & standard 2 drilling & 254.2 & 838.8 \\
\hline Drilling 6 holes $\emptyset 10$ & standard 1 drilling & 253.8 & 837.6 \\
\hline Drilling 15 holes $\emptyset 8$ & standard 2 drilling & 369.9 & 1220.8 \\
\hline freis main shaft cover & horizontal 2 milling & 314.7 & 769.0 \\
\hline drill 6 holes main shaft cover & standard 2 drilling & 252.3 & 832.7 \\
\hline drilling 6 holes secondary shaft cover & standard 1 drilling & 251.9 & 831.4 \\
\hline \multicolumn{2}{|l|}{ LH-Total } & 30331.0 & 26817.8 \\
\hline \multicolumn{2}{|l|}{ LH-RH Total } & 66053.4 & 58183.3 \\
\hline
\end{tabular}


Table 4. Assembly Operation List of Design 1

\begin{tabular}{|l|c|c|c|}
\hline \multicolumn{4}{|c|}{ Gear Transmission Box LH } \\
\hline Assy Operation & Machine & $\begin{array}{c}\text { Machine } \\
\text { cost (Rp) }\end{array}$ & $\begin{array}{c}\text { labour } \\
\text { cost (Rp) }\end{array}$ \\
\hline welding main body B-C Assy & CO new K200 855502 weld & 1171.8 & 2894.6 \\
\hline welding main body A-(BC) Assy & CO new K200 855503 weld & 1052.3 & 2601.1 \\
\hline welding 6 part tube support Assy & CO new K200 855502 weld & 308.5 & 757.5 \\
\hline \multicolumn{3}{|c|}{ LH-Total } & \multicolumn{2}{c|}{8785.59} \\
\hline \multicolumn{3}{|c|}{ Gear Transmission Box RH } & Machine \\
\hline Assy Operation & Machine & $\begin{array}{c}\text { labour } \\
\text { cost (Rp) }\end{array}$ \\
\hline welding main body B-C Assy & CO new K200 855502 weld & 1171.8 & 2894.6 \\
\hline welding main body A-(BC) Assy & CO new K200 855503 weld & 1052.3 & 2601.1 \\
\hline welding 6 part tube support Assy & CO new K200 855502 weld & 308.5 & 757.5 \\
\hline \multicolumn{2}{|c|}{ LH-Total } & 8785.59 \\
\hline \multicolumn{2}{|c|}{ LH-RH Total } & 17571.2 \\
\hline
\end{tabular}

Table 5 below show us that using equation (18) Forming operations in Design 1 can be done. $\left(e_{u}=0.1654\right.$, Subekti, 2004)

Table 5. Strain factor of Design 1

\begin{tabular}{|l|c|c|c|}
\hline \multicolumn{1}{|c|}{ part name } & strain factor & $e_{u}$ & conclution \\
\hline main shaft cover & 0.097631073 & 0.1654 & no failure \\
\hline pulley shaft cover & 0.140079366 & 0.1654 & no failure \\
\hline secondary shaft cover & 0.033795371 & 0.1654 & no failure \\
\hline
\end{tabular}

Manufacturing cost is calculated by summing material, machine of production, labour of production, assembly and overhead cost. Over head cost was calculated similarly method with initial design. material cost is accumulated from plates cost, welding filler and tube cost. Assembly cost is sum of machine cost and labour cost in assembly operation.

Table 6. Manufacturing cost of design 1

\begin{tabular}{|c|c|c|c|c|c|c|}
\hline part name & $\begin{array}{c}\text { material } \\
\text { cost }\end{array}$ & $\begin{array}{c}\text { machine } \\
\text { cost }\end{array}$ & $\begin{array}{c}\text { labour } \\
\text { cost }\end{array}$ & $\begin{array}{c}\text { assembly } \\
\text { cost }\end{array}$ & $\begin{array}{c}\text { Overhead } \\
\text { cost }\end{array}$ & $\begin{array}{c}\text { manufacturing } \\
\text { cost }\end{array}$ \\
\hline GTB LH & $89,931.2$ & $35,722.5$ & 31365.6 & 8785.59 & $12,083.9$ & $177,888.8$ \\
\hline GTB RH & 87346.34 & $30,331.0$ & $26,817.8$ & 8785.59 & $10,960.7$ & $164,241.4$ \\
\hline total & $177,277.6$ & $66,053.4$ & $58,183.3$ & $17,571.2$ & $23,044.7$ & $342,130.2$ \\
\hline
\end{tabular}

\section{Design 2}

Design 2, as like as design 1 using ST 37 material. But there is deference in main body. In this design, main body consist of a part that. Thickness of main body $3.2 \mathrm{~mm}$. there is supporting plate $8 \mathrm{~mm}$ thickness in every side of GTB. This design consists of 11 parts in a side. (shown in Figure 4)

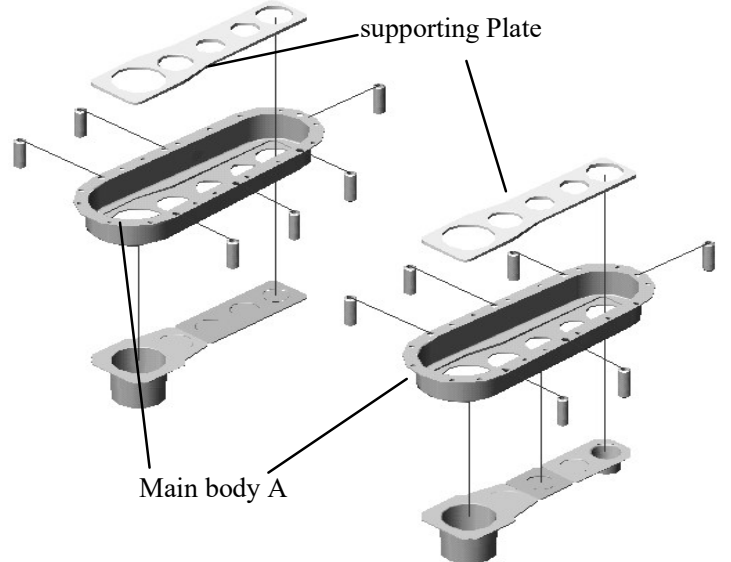

Figure 5. Design 2 of GTB breakdown
In this design similarly design 1 , GTB are produced in combination metal forming, welding and machining too. There are 5 metal forming operations, 16 machining and 2 assembly operations in GTB LH. And in other side there are 4 metal forming operations, 12 machining and 2 assembly operations. All of them are shown in table 7 and 8 below.

Table 7. Production operation list of design 2

\begin{tabular}{|c|c|c|c|}
\hline \multicolumn{4}{|c|}{ Gear Transmission Box LH } \\
\hline Operation & Machine & $\begin{array}{l}\text { Machine } \\
\text { cost }(\mathrm{Rp})\end{array}$ & $\begin{array}{c}\text { labour } \\
\text { cost (Rp) }\end{array}$ \\
\hline main body forming & hidrolic pressure & 5839.1 & 2113.5 \\
\hline plate support foming & hidrolic pressure & 5368.8 & 1943.3 \\
\hline main shaft cover forming & hidrolic pressure & 4773.4 & 1727.8 \\
\hline secondary shaft cover (LH) forming & hidrolic pressure & 4330.2 & 1567.4 \\
\hline pulley shaft cofer forming & hidrolic pressure & 4712.5 & 1705.7 \\
\hline tube support cutting & band SAW Mega & 659.3 & 2755.1 \\
\hline Full side freis mill & vertical milling & 1903.4 & 5167.4 \\
\hline Drilling 2 pen holes & standard 1 drilling & 827.4 & 2730.6 \\
\hline Full side freis mill & vertical milling & 411.8 & 1118.0 \\
\hline Boring+chamfering 5 holes together & horizontal 2 milling & 469.0 & 1146.1 \\
\hline Drilling oil taping & radial drilling & 216.7 & 715.1 \\
\hline Drilling oil sprue & radial drilling & 216.7 & 715.1 \\
\hline Drilling 8 holes $\varnothing 7$ & standard 1 drilling & 290.5 & 958.8 \\
\hline Drilling 6 holes $\emptyset 8$ & standard 2 drilling & 258.6 & 853.5 \\
\hline Drilling 6 holes $\emptyset 10$ & standard 1 drilling & 251.9 & 831.4 \\
\hline Drilling 15 holes $\emptyset 8$ & standard 2 drilling & 365.5 & 1206.1 \\
\hline freis main shaft cover & horizontal 2 milling & 314.7 & 769.0 \\
\hline freis pulley shaft cover & horizontal 2 milling & 296.1 & 723.7 \\
\hline drill 6 holes main shaft cover & standard 2 drilling & 252.3 & 832.7 \\
\hline drilling 3 holes secondary shaft cover & standard 1 drilling & 213.3 & 704.1 \\
\hline Drilling 6 holes pulley shaft cover & standard 2 drilling & 252.3 & 832.7 \\
\hline \multicolumn{2}{|l|}{ LH-total } & 32223.5 & 31117.0 \\
\hline \multicolumn{4}{|c|}{ Gear Transmission Box RH } \\
\hline Operation & Machine & $\begin{array}{l}\text { Machine } \\
\text { cost }(\mathrm{Rp})\end{array}$ & $\begin{array}{c}\text { labour } \\
\text { cost (Rp) }\end{array}$ \\
\hline main body forming & hidrolic pressure & 5839.1 & 2113.5 \\
\hline plate support foming & hidrolic pressure & 5368.8 & 1943.3 \\
\hline main shaft cover forming & hidrolic pressure & 4773.4 & 1727.8 \\
\hline secondary shaft cover $(\mathrm{RH})$ forming & hidrolic pressure & 4621.2 & 1672.7 \\
\hline tube support cutting & band SAW Mega & 659.3 & 2755.1 \\
\hline Full side freis mill & vertical milling & 1903.4 & 5167.4 \\
\hline Drilling 2 pen holes & standard 1 drilling & 827.4 & 2730.6 \\
\hline Full side freis mill & vertical milling & 411.8 & 1118.0 \\
\hline Boring+chamfering 5 holes together & horizontal 2 milling & 469.0 & 1146.1 \\
\hline Drilling 6 holes $6 \varnothing 7$ & standard 1 drilling & 258.6 & 853.5 \\
\hline Drilling 6 holes $\emptyset 8$ & standard 2 drilling & 258.6 & 853.5 \\
\hline Drilling 6 holes $\varnothing 10$ & standard 1 drilling & 251.9 & 831.4 \\
\hline Drilling 15 holes $\varnothing 8$ & standard 2 drilling & 365.5 & 1206.1 \\
\hline freis main shaft cover & horizontal 2 milling & 314.7 & 769.0 \\
\hline drill 6 holes main shaft cover & standard 2 drilling & 252.3 & 832.7 \\
\hline drilling 3 holes secondary shaft cover & standard 1 drilling & 251.9 & 831.4 \\
\hline \multicolumn{2}{|l|}{$\mathrm{RH}$ - total } & 26826.8 & 26552.1 \\
\hline \multicolumn{2}{|c|}{ RH-LH Total } & 59050.4 & 57669.1 \\
\hline
\end{tabular}

Table 8. Assembly operation list of design 2

\begin{tabular}{|c|c|c|c|}
\hline \multicolumn{4}{|c|}{ Gear Transmission Box LH } \\
\hline Assy Operation & Machine & $\begin{array}{l}\text { Machine } \\
\text { cost (Rp) }\end{array}$ & $\begin{array}{c}\text { labour } \\
\text { cost }(R p)\end{array}$ \\
\hline welding plate support Assy & CO new K200 855502 welding & 520.6 & 1297.3 \\
\hline welding 6 part tube support Assy & CO new K200 855503 welding & 294.7 & 757.5 \\
\hline \multicolumn{2}{|c|}{ LH-total } & \multicolumn{2}{|c|}{2870.1} \\
\hline \multicolumn{4}{|c|}{ Gear Transmission Box RH } \\
\hline Assy Operation & Machine & $\begin{array}{l}\text { Machine } \\
\text { cost (Rp) }\end{array}$ & $\begin{array}{c}\text { labour } \\
\text { cost }(\mathrm{Rp})\end{array}$ \\
\hline welding plate support Assy & CO new K200 855502 welding & 520.6 & 1297.3 \\
\hline welding 6 part tube support Assy & CO new K200 855503 welding & 294.7 & 757.5 \\
\hline \multicolumn{2}{|c|}{$\mathrm{RH}$ - total } & \multicolumn{2}{|c|}{$\frac{1}{2870.1}$} \\
\hline \multicolumn{2}{|c|}{ RH-LH Total } & \multicolumn{2}{|c|}{5740.2} \\
\hline
\end{tabular}


All plate metal forming operation can be done and they aren't caused crack and failure. (Table 9) all of strain factor are lower than elongation unaxial.

\section{Table 9. Strain factor of design 2}

\begin{tabular}{|l|c|c|c|}
\hline \multicolumn{1}{|c|}{ part name } & strain factor & $e_{u}$ & conclution \\
\hline main body & 0.143575107 & 0.1654 & no failure \\
\hline main shaft cover & 0.097631073 & 0.1654 & no failure \\
\hline pulley shaft cover & 0.140079366 & 0.1654 & no failure \\
\hline secondary shaft cover & 0.033795371 & 0.1654 & no failure \\
\hline
\end{tabular}

Manufacturing cost of design 2 are shown in Table 10 below;

Table 10. Design 2 Manufacturing cost

\begin{tabular}{|c|c|c|c|c|c|c|}
\hline part name & $\begin{array}{c}\text { material } \\
\text { cost }\end{array}$ & $\begin{array}{c}\text { machine } \\
\text { cost }\end{array}$ & $\begin{array}{c}\text { labour } \\
\text { cost }\end{array}$ & $\begin{array}{c}\text { assembly } \\
\text { cost }\end{array}$ & $\begin{array}{c}\text { overhead } \\
\text { cost }\end{array}$ & $\begin{array}{c}\text { manufacturing } \\
\text { cost }\end{array}$ \\
\hline GTB LH & $70,552.9$ & $32,223.5$ & $31,117.0$ & $2,870.1$ & $10,148.7$ & $146,912.2$ \\
\hline GTB RH & $67,968.0$ & $26,826.8$ & $26,552.1$ & $2,870.1$ & $9,023.3$ & $133,240.3$ \\
\hline Total & $138,520.9$ & $59,050.4$ & $57,669.1$ & $5,740.2$ & $19,172.0$ & $280,152.5$ \\
\hline
\end{tabular}

\section{DISCUSSION}

In cost parameter, the biggest reduction of two design for initial design are in material cost. Even design 2 reduces material cost till $51.9 \%$. Initial Design manufacturing cost is $\mathrm{Rp} 387,671.7$. Design 1's is Rp 342,130.2. Design 2's is Rp 280,152.5. Overall, Design 2 performs the lowest cost between the alternatives. It reduces total manufacturing cost until $27 \%$, it is bigger than Design 1 that only $11.75 \%$ (figure 6 ).

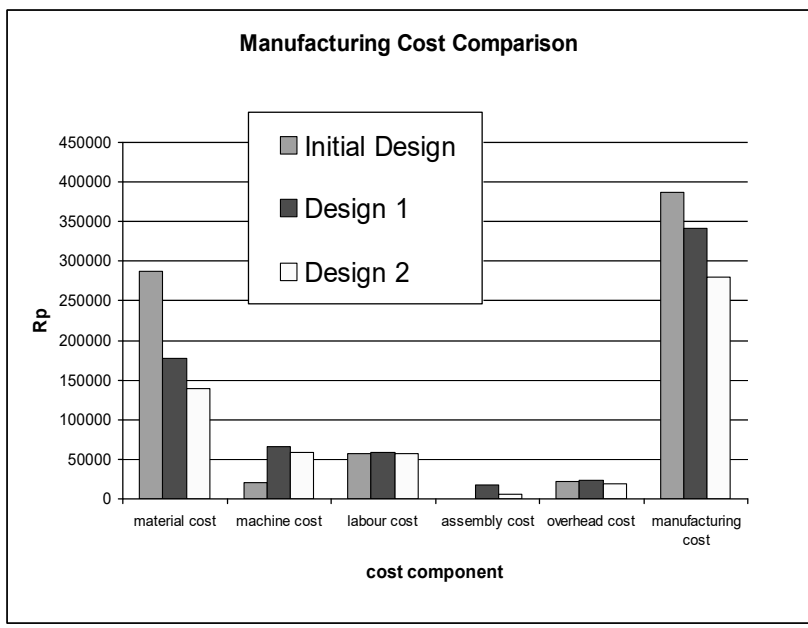

Figure 6. Manufacturing cost comparison chart

About the loads that are detained by GTB, maximum load $\left(\mathrm{F}_{\mathrm{m}}\right)$ is $57.9 \mathrm{kN}$ or 5,7 ton. Smallest longitudinal section wide of design 2 are in plate support. The size is;

$$
\begin{aligned}
\mathrm{L} & =8 \text { (thickness) } \times 90 \text { (width) } \\
& =720 \mathrm{~mm}^{2} .
\end{aligned}
$$

With yield strength (technique) of ST $37=366.6$ $\mathrm{N} / \mathrm{mm}^{2}$ (Subekti, 2004) and safety factor $10 \%$, maximum load that can be detained by desain 2 is

$$
\begin{aligned}
\mathrm{F}_{\text {platemax }} & =720 \times(1-10 \%) \times 366.6 \\
& =237556.8 \mathrm{~N}=237.6 \mathrm{kN}
\end{aligned}
$$

Because of $F_{\text {platemax }}>F_{m}$, technically Design will be safe.

About the investment, company needn't buy pressure machine for metal forming operation. Using equation (13), Maximum force to forming is 497 ton (piercing plate support). Company have hydraulic pressure machine, with the specification below;

Machine name : MINCANG type MCD $75 \mathrm{Hp}$

Dimension : $6700 \times 4650 \times 3800 \mathrm{~mm}$

Capacity $: 500$ ton $/ 8 \mathrm{~mm}$

Power $\quad: 75 \mathrm{Hp}-380$ Volt $-50 \mathrm{~Hz}$

Pressure $\quad: 210 \mathrm{Kg} / \mathrm{cm} 2$

Investment is needed to make dies of pressure machine for metal forming. It is about $14-15$ milion rupiahs or US\$1,522.- US\$ 1,630 (with 1 US\$ = Rp 9,200.-) for five dies. How small amount of it if compared with improvement that was resulted. This methods could be done by the company because the aplication of this design will affect positively to a whole process.

\section{REFERENCES}

Anderson, D.M., (2007). 'Design for Manufacturability'.Design for Manufacturability and Concurrent Engineering. www.design4manu facturability.com. 5 Oktober 2007

Andrews, R.R., (2006). Design with Manufacturing in Mind. www.foster-miller.com. 1 Agustus 2007 pp 3

Deutchman, A.D., (1975). Machine Design Theory and Practice. New York. Macmillan Publishing Co.,Inc. pp 540-544 \& 788-792

Kalpakjian. (1989). Manufacturing Engineering Technology. Addison Wesley: USA pp 240

Rao, P.N., Maufacturing Technology, Foundry, Forming and Welding $2^{\text {nd }}$ Edition, McGraw-Hill.

Gautomo, S., (2004) Perancangan Press Tool bearing Case mesin Pemotong rumput PT Agrindo Gresik, Final Project, ITS Surabaya. pp 46-49

Rochim, T., (1993). Teori Dan Teknologi Proses Pemesinan. Bandung. Laboratorium Teknik Industri FTI - ITB pp 18-21

Ulrich, Karl T. and Eppinger, Steven D. (2000) Product Design and Development 2nd Edition. London. McGraw-Hill Co.,Inc pp 221-244 